\title{
Topinambur - new possibilities for use in a supplementation diet
}

\author{
Aleksandra Szewczyk ${ }^{1, D \oplus}$, Mirosław Zagaja ${ }^{1, C \oplus}$, Jarosław Bryda ${ }^{1, C \oplus}$, Urszula Kosikowska ${ }^{2, C} \oplus$, \\ Dagmara Stępień-Pyśniak ${ }^{3, C}{ }^{\oplus}$, Stanisław Winiarczyk ${ }^{3, C} \oplus$, Marta Andres-Mach ${ }^{1, E \oplus}$ \\ 1 Institute of Rural Health, Lublin, Poland \\ ${ }^{2}$ Medical University, Lublin, Poland \\ ${ }^{3}$ University of Life Sciences, Lublin, Poland \\ A - Research concept and design, B - Collection and/or assembly of data, C - Data analysis and interpretation, \\ $D$ - Writing the article, $E$ - Critical revision of the article, F - Final approval of article
}

Szewczyk A, Zagaja M, Bryda J, Kosikowska U, Stępień-Pyśniak D, Winiarczyk S, Andres-Mach M. Topinambur - new possibilities for use in a supplementation diet. Ann Agric Environ Med. 2019; 26(1): 24-28. doi: 10.26444/aaem/102767

\begin{abstract}
Jerusalem artichoke (Helianthus tuberosus Linne) is a tuberous perennial plant of the Asteraceae family, which originates from North America, and is also known as wild sunflower or topinambur (TPB). It is characterized by good tolerance to frost, drought and poor soil, strong resistance to pests and plant diseases. For ages it was cultivated due to being both an edible tuber and having healing properties. In folk medicine, TPB leaves are used for the treatment of bone fractures and pain. TPB tubers are rich in sugar and have therefore been used for the production of functional food ingredients, such as inulin. Moreover, TPB is one of the potential crops for bioenergy production, such as bioethanol, biobuthanol and biodiesel, and chemicals (lactic acid, butyric acid). A number of bioactive compounds from the above ground parts of this plant have been isolated which have demonstrating antifungal, antioxidant and anticancer activities. In recent years, a number of animal experiments have been carried out to assess the health properties of TPB. Obtained results show that TPB possess a wide spectrum of medical applications, e.g. reduction in the levels of plasma glucose, total cholesterol and triglyceride. Interestingly, TPB has been shown to be a valuable alternative source of prebiotic compounds. This review article presents recent scientific reports on the chemical and biological properties of TPB and its potential use as a prebiotic diet supplement.
\end{abstract}

\section{Key words}

Topinambur, microbiota, prebiotics, biofuels

Characteristics of TPB. TPB is a perennial herbaceous plant of the Asteraceae family, genus Helianthus. Helianthus tuberosus belongs to the same genus as sunflower (H. annuus). It is an old species which originates from North America [1] and in the $17^{\text {th }}$ century was brought to Europe where it grows naturally [2]. Today, it is widely cultivated in many countries. TPB develops underground stolons forming shaped tubers similar to potatoes. The colours of the tubers range from pale brown to white, red and purple [3].

TPB can be developed under different environmental conditions because it is characterized by good tolerance to frost, drought and poor soil. Moreover, cultivation of wild sunflower does not need too much management and protection due to its strong resistance to pests and plant diseases [3,4]. Its fast growth is a good natural control against weeds [5].

TPB produces sugars in the above ground parts of the plant and stores them in the roots and tubers. Tubers consists of water (75-79\%), carbohydrates (15-16\%), proteins (2-3\%) and fat (2-3\%). Additionally, other microelements such as phosphorus $(\mathrm{P})$, potassium $(\mathrm{K})$, calcium $(\mathrm{Ca})$, magnesium $(\mathrm{Mg})$ and ferrum $(\mathrm{Fe})$ are present and constitute $0.5 \%$ of the fresh weight. Tubers also contain vitamins such as vitamin $C$ and $\mathrm{A}$ [6]. Among the carbohydrates found in tubers, inulin can constitute as much as $80 \%$ or more. The remaining sugars are in the form of fructose, sucrose, and glucose [7].

Address for correspondence: Aleksandra Szewczyk Institute of Rural Health, Jaczewskiego 2, 20-090, Lublin, Poland

e-mail: olciak1892@gmail.com

Received: 30.11.2018; accepted: 11.01.2019; first published: 06.02.2019
Inulin is a storage polysaccharide found in many plants and classified as fructans. Chemically, it is a linear biopolymer of $D$-fructose units connected by $\beta(2,1)$ glycosidic linkages that are terminated by $\mathrm{D}$-glucose molecules bonded to fructose by $(2 \rightarrow 1)$ a-bond (Fig. 1) [8]. The degree of polymerization

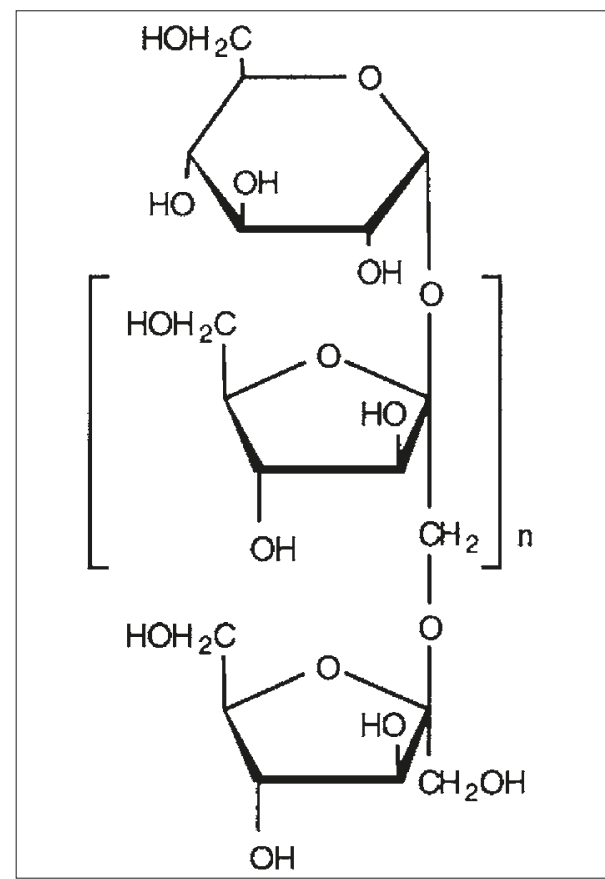

Figure 1. Chemical formula of an inulin 
of inulin varies according to species, cultivar, production conditions, physiological age, among other factors, and generally ranges from $2-60$ fructose residues. TPB and chicory have an inulin content of $150 \mathrm{~g} \mathrm{~kg}-1$ fresh weight basis, and $750 \mathrm{~g} \mathrm{kg-1}$ dry weight basis [8, 9]. Inulin is also present in vegetables such as leeks and onions, as well as in fruits such as banana. This D-fructose polymer is a water soluble fibre, which can initially be found in high levels in the stems which, by the end of the growing cycle is transferred to the tubers [6].

Inulin, apart from being an important reserve material found in plants, is also a natural prebiotic. Prebiotics are defined as non-digestible food ingredients that selectively stimulate the growth of probiotic bacteria in the gut [10]. Prebiotics confer many health benefits on the gastrointestinal tract, immune system and brain function, as well as increasing absorption of minerals, regulating blood lipids, and reducing cancer risk [11]. Among the widely-known prebiotics, inulintype fructans are the most commonly used.

Inulin is indigestible by the human small intestinal digestive enzymes but is fermented by certain bacteria (e.g. Bifidobacterium and Lactobacillus) in the large intestine by lactate and short-chain fatty acids (SCFAs) [12]. Specific types of prebiotics, such as inulin and fructooligosaccharides (FOS), have been shown to stimulate Bifidobacterium growth and to reduce harmful bacteria in the human colon [13]. FOS and inulin have also been reported as immune modulating agents [14].

Multiple applications of TPB. For decades, TPB has been used as green or brackish fodder for animals [15]. TPB is mainly cultivated for the production of functional food ingredients, such as soluble fibre (inulin), oligofructose and fructose $[16,17]$. TPB is one of the potential crops for bioenergy production, e.g. bioethanol, methane and biogas [18]. Interestingly, it is possible to extract some bioactive compounds from TPB stems and leaves which are used in the pharmaceutical sector $[19,20]$. In folk medicine, TPB leaves are used for the treatment of bone fractures, skin wounds, swelling and pain [3]. Moreover, a number of bioactive compounds demonstrating antifungal, antioxidant and anticancer activities have been isolated from the aboveground parts of the plant $[3,19-24]$. Due to the high content of inulin, TPB represents a valuable alternative source of prebiotic compounds [25]. Various possibilities of using TPB are presented in Figure 2.

Biofuels. Due to the high content of inulin, TPB is one of the potential crops for bioenergy production, such as bioethanol, biobuthanol and biodiesel. Inulin found in TPB tubers can be

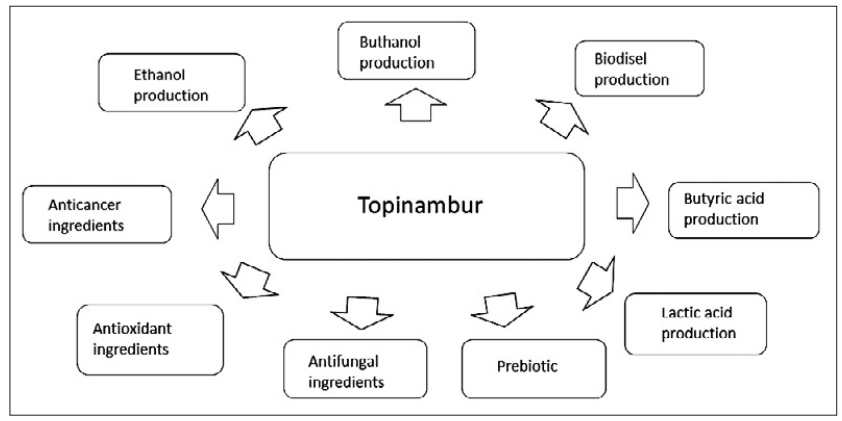

Figure 2. Multiple applications of TPB easily converted by using different technologies. For decades, TPB has been used for ethanol production. Interestingly, in $19^{\text {th }}$ century TPB tubers were used for beer production in France [26].

There are two different methods for bioethanol production from TPB. The first involves inulin hydrolysis and sugar fermentation. Typically, inulin from TPB tubers needs to be hydrolyzed into fermentable sugars (fructose and glucose) by mineral acids or inulinase enzyme. Next, sugar is fermented into ethanol by using yeasts. The most commonly used strains in alcohol production are Saccharomyces cerevisiae and Zymomonas mobilis. During this process, the fermentable sugar loss caused by separation and transfer of sugars from hydrolyzer into fermenter is observed. The second method for bioethanol production from TPB is characterized by simultaneous inulin hydrolysis and sugar fermentation. This process is carried out in one bioreactor using a mixture of inulinase or mineral acids and yeast. In contrast to the first method, in this process significant reduction of the fermentable sugar loss is observed. Importantly, a direct conversion of inulin into ethanol decreases the production costs [3].

TPB tubers have also found application in the production of biodiesel. In research carried out by Cheng et al. [27] on hydrolysate of TPB, tubers were used as the source of carbon for lipids accumulation by Chlorella protothecoides. Next, lipids were extracted and converted into biodiesel through the chemical reactions of transesterification. In subsequent studies, Sung et al. [28] used TPB tubers for lipid production by yeast Cryptococcus sp. under the following reaction condition $-117^{\circ} \mathrm{C}$ for $49 \mathrm{~min}$. At this optimal condition, $1.73 \mathrm{~g} / \mathrm{L} / \mathrm{d}$ of lipid was produced in a day, which is more than by using the defined medium containing pure fructose as a substrate. Obtained results suggest that TPB tubers are a good and cheap substrate for biodiesel production.

Inulin from TPB is also used for biobutanol production. Research carried out by Sarchami and Rehmann [29] focused on optimizing enzymatichydrolysis of TPB tubers to maximize inulin conversion, and further fermentation of hydrolyzate to butanol by Clostridium saccharobutylicum DSM 13864. Obtained results indicate that the experimental range for inulin conversion was a temperature of $48^{\circ} \mathrm{C}, \mathrm{pH}$ of 4.8 , and substrate concentration of $60 \mathrm{~g} / \mathrm{L}$. C. saccharobutylicum DSM 13864 used $45.6 \mathrm{~g} / \mathrm{L}$ sugar to produce $9.6 \mathrm{~g} / \mathrm{L}$ butanol. The yield and the acetone-butanol-ethanol concentration was $0.33 \mathrm{~g}_{\text {solvent }} / \mathrm{g}_{\text {sugar }}$ and $14.9 \mathrm{~g} / \mathrm{L}$, whereas in a study by Chen et al. [30] Clostridium acetobutylicum L7 was able to ferment enzymatic hydrolyzate of TPB. The culture used $62.9 \mathrm{~g} / \mathrm{L}$ sugars to produce $11.21 \mathrm{~g} / \mathrm{L}$ butanol, resulting in a yield of $0.29 \mathrm{~g}_{\text {solvent }} / \mathrm{g}_{\text {sugar }}$.

Chemicals. The next significant use for TPB has been found in generating chemicals, such as lactic acid and butyric acid. Lactic acid has been employed in many fields, such as the food, pharmaceutical and chemical industries. Andersen and Greaves were the first to use TPB tubers to produce D-lactic acid [31]. Ge et al. [32] used mixed culture of Aspergillus niger and Lactobacillus sp to produce L-lactic acid in the fermentation process. The maximum L-lactic acid concentration of $120.5 \mathrm{~g} / \mathrm{L}$ was obtained after $36 \mathrm{~h}$ of fed-batch fermentation. Wang et al. [33] used TPB powder to obtain high-optical purity of L-lactate using a thermophilic Bacillus coagulans strain. The final product concentration 
of $134 \mathrm{~g} / \mathrm{L}$ was obtained and the optical purity was $99 \%$. In addition to the production of lactic acid, TPB is also used in the preparation of the butyric acid. In studies carried out by Huang et al. [34], TPB hydrolysates were used to produce butyric acid by immobilized Clostridium tyrobutyricum during fed-batch fermentation, where the maximum concentration of butyric acid obtained was $60.4 \mathrm{~g} / \mathrm{L}$. TPB also has potential for generating other chemicals, such as citric acid [35], succinic acid [36] and sorbitol [37].

Bioactive compounds. In addition to the wide use of TPB tubers, the leaves also have important applications. They are used, for example, in folk medicine for the treatment of bone fractures, skin wounds, swelling and pain [3]. It has been shown that bioactive compounds isolated from the above ground parts of TPB have an positive effect on health. Phenolic acid isolated from TPB leaves have antifungal activities [22], while chlorogenic and isochlorogenic acids have good antioxidant properties [20]. Moreover, ethyl acetate has activities against breast cancer, lung cancer and epithelial cervical cancer [21]. In studies carried out by Liu et al. [22], the antifungal properties of TPB leaves extracts was studied against several fungi, such as Rhizoctonia solani, Gibberella zeae, Alternaria solani and Botrytis cinerea. To extract TPB leaves, several different solvents were used (petroleum ether, ethyl ether, ethyl acetate and water). Obtained results suggest that TPB leaf extracts exerted different antifungal activities, depending on the solvent. The inhibitory effects of extracts of organic solvents were significantly higher than those of aqueous extracts. Similarly, results of other studies conducted by Chen et al. [24] proved that three out of six phenolic acids (caffeic acid, 3,4-dicaffeoylquinic acid and 1,5-dicaffeoylquinic acid) isolated from TPB leaves were identified as being responsible for Gibberella zeae inhibition.

TPB studies in animal experiments. Considering the varied spectrum of TPB applications in both industry and widelyunderstood medicine, in recent years, researchers have started investigating the potential use of TPB as a dietary supplement in many diseases. One of the first stages of the research was to assess the potential therapeutic properties of new substances by using animal models.

Diabetes. Taking into account the significant influence of TPB and inulin diet on the regulation of glucose levels, a number of animal experiments have been carried out to assess the health properties of TPB in animal models of diabetes. Zacky et al. [38] investigated the biological effects of TPB tubers on glycaemic response in diabetic rats. Obtained results indicated that a diet containing TPB tubers reduced serum glucose levels, total cholesterol, LDL cholesterol and triglycerides in hyperglycaemic rats, compared to the positive control. In addition, improvements in liver and kidney functions were observed, which may have been the result of the TPB tubers added to the diet.

Abdel-Hamid et al. [39] investigated anti-fibrotic effects of TPB tubers by biochemical analysis of liver enzymes activities and total bilirubin levels. Obtained results suggest that TPB treatment has a promising hepatoprotective effect against carbon tetrachloride (CCl4)-induced fibrosis via modulation of apoptotic signaling and fibrogenic activity. Results obtained by Wang et al. [40], using streptozotocin (STZ)-induced diabetic rats, indicated that treatment with
TPB has potentially positive effects in the relief of symptoms of diabetes by repairing the liver damage caused by STZ. Similar to TPB, chungkookjang (CKJ; fermented soybeans) has also been considered to modulate energy and glucose metabolism. Yang et al. [41] evaluated an anti-diabetic effects of TPB and CKJ supplementary diet in diabetic rats. Animals were divided into four experimental groups (diabetic-control, $\mathrm{CKJ}, \mathrm{CKJ}+\mathrm{TPB}$, and TPB). CKJ, CKJ+TPB and TPB groups were fed for eight weeks on experimental diets containing $5 \%$ lyophilized CKJ, 5\% lyophilized CKJ+5\% lyophilized TPB, or $5 \%$ lyophilized TPB, respectively. The diabetic-control group had diet without CKJ and TPB. Obtained results indicated that TPB improved insulin sensitivity, whereas $\mathrm{CKJ}$ potentiated glucose-stimulated insulin secretion and enhanced $\beta$-cell function in the pancreas. Chang et a. [42] investigated the molecular mechanisms and physiological responses of the TPB supplementation in rats in the prevention of type 2 diabetes and non-alcoholic fatty liver disease via a transcriptome analysis. Results obtained indicated that a diet containing $10 \%$ TPB significantly reduced the expression of genes malic enzyme $1(\mathrm{Mel})$, decorin (Dcn) and nicotinamide phosphoribosyltransferase (Nampt), whereas fructose feeding increased the expression of these genes. Moreover, rats in the group containing fructose in the diet demonstrated hepatic triacylglycerol) accumulation and hepatic steatosis, whereas TPB supplementation significantly decreased these changes.

TPB as prebiotic. Gut microbes play an important role in the health and disease of the host. Prebiotics are nondigestible fibre substances used as a substrate for beneficial bacteria which promote the growth of intestinal bacteria. As mentioned above, inulin is one of the most common natural prebiotics used as a dietary supplement, and TPB is one of the main sources for inulin from higher plants. [43]. Due to the high content of inulin, TPB also represents a valuable alternative source of prebiotic compounds [25]. This property of TPB was used in studies carried out by Samal et al. [44]. The aim of their experiment was to investigate the effect of dietary supplementation with TPB tuber powder as a prebiotic on the growth of probiotic bacteria in the gut, as well as its growth performance and nutrient digestibility in rats. Animals were on a 12 -week diet supplemented with 0 , 2,4 and $6 \%$ of TPB tubers. Obtained results indicated that the addition of TPB to the diet did not affect feed intake nor the growth performance of rats. Moreover, the apparent absorption of minerals such as calcium and phosphorus was observed. Diet supplementation with TPB tuber powder increased the population of Lactobacillus spp. and Bifidobacterium spp. microbiota in the caecal, colonic and rectal digesta. Valdovska et al. [45] investigated the effect of TPB and probiotics on the gut microbiota of weaning pigs. Animals were fed for five weeks on an experimental diet supplemented with TPB and two probiotics (Lactobacillus reuteri and Pediococcus pentosaceus) in different doses. Obtained results demonstrated that supplementing the diet with both probiotics and TPB (5\% of basal diet) improved the levels of faecal Lactobacillus, and reduced the levels of enteric pathogens in faecal samples, compared with the control group. Moreover diet supplementation with TPB alone significantly increased the regeneration process in the intestine of pigs. Boonanuntanasarn et al. [46] investigated the effects of dietary supplementation with inulin and TPB on intestinal microbiota of Nile tilapia fingerlings. Nile 
tilapia larvae were fed for 12 weeks on experimental diets supplemented with inulin at as dose of 2.5 and $5.0 \mathrm{~g} / \mathrm{kg}$, and $\mathrm{TPB}$ at 5.0 and $10.0 \mathrm{~g} / \mathrm{kg}$ from the first feeding. Obtained results demonstrated that in fish fed a dietary supplementation of $5.0 \mathrm{~g} / \mathrm{kg}$, inulin and TPB at both levels had higher lactic acid bacteria counts and Bifidobacterium spp. compared to fish fed the basal diet.

Results obtained from the last preliminary screening experiments conducted by the authors of the current study also confirmed the additive impact of TPB supplementary diet on the probiotic bacteria in the gut of mice (data not published). All experiments were performed on adolescent male Albino Swiss mice. Animals were divided into three experimental groups (TPB, Inulin and Control). According to the manufacturer's recommended daily intakes, mice received TPB (Organic, Sieniawa, Poland, $250 \mathrm{mg} / \mathrm{kg}$ ), inulin $(66 \mathrm{mg} / \mathrm{kg})$ via oral administration No side-effects on body weight, food intake or behaviour were observed. To evaluate any changes in gut microbiota, microbiological analysis was performed of the faecal samples from each group by inoculating on petri dishes with selective media. Bacterial counts were recorded as colony-forming units $(\mathrm{CFU}) / \mathrm{ml}$. Obtained results showed that TPB stimulated growth of one of the most common probiotic bacteria - Lactobacillus gasseri, as well as the Enterobacteriaceae family (Escherichia coli, Enterobacter asburiae, Kliebsiella oxytoca (Fig. 3).

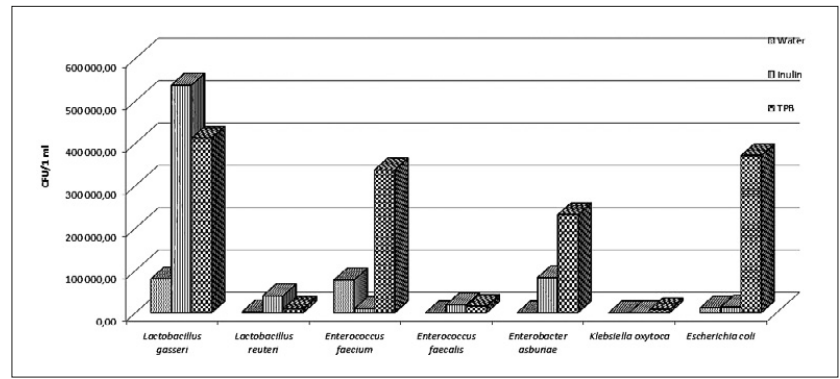

Figure 3. Impact of TPB supplementary diet on intestinal microbiota in mice. Routine media used for culturing and subculturing examined isolates included TSA (Tripticasein Soy LAB-AGAR, Biocorp), TSB (Tripticasein Soy BROTH, Biocorp), MRS (MRS LAB-AGAR, Biocorp), Mueller-Hinton agar (Bio-Rad) with blood, Sabouraud (Sabouraud Dextrose LAB-AGAR, Biocorp). Semi-quantitative results were read after incubation in conditions proper for both aerobic and anaerobic bacteria, as well as for fungi. For each morphotype, the semi-quantitative colony counts were calculated by the number of colonies visible on the agar plate (or on the part of the agar plate in the case of very high growth of microorganisms) in relation to the dilution $(100 \times$ or $1000 x)$, and results reported as colony-forming units per milliliter (CFU/ml). Isolates identification included colony morphology and mass spectrometry protein profiles analysis by matrix-assisted laser desorptionionization time-of-flight mass spectrometry (MALDI-TOF MS), using a MALDIbiotyper (Bruker Daltonik, Germany)

Due to the fact that only preliminary screening studies were carried out, it would be beneficial to conduct studies on a larger group of animals to avoid high standard deviations, and to obtain statistically significant results. However, a tendency was noted for an increased amount of microbiota in the studied animals. This may undoubtedly indicate a positive effect of the TPB diet on gut microbiota, and thus on the proper functioning of intestinal microbiome homeostasis.

\section{CONCLUSIONS}

TPB is a potential crop for bioenergy production - bioethanol, biobuthanol and biodiesel, as well as chemicals. Bioactive compounds isolated from above ground parts of TPB have a positive effect on health, with antifungal, antioxidant and anticancer properties. Additionally, TPB possesses a wide spectrum of medical applications, such as in the reduction of plasma glucose or total cholesterol and triglyceride levels.

The problem of a properly balanced diet affects most of the population and is mainly caused by the wide availability of processed food. Inadequate diet leads to metabolic disorders, which consequently cause a number of diseases of the digestive system, as well as disorders of the nervous system, according to the latest research.

Since it is known that the intestines play the role of a second "brain" in the body, scientists are conducting intensive research in the search for new natural supplements that will positively affect the proper functioning of the intestines. According to the results of recent research, TPB has all the predispositions to be included in the group of very valuable natural supplements that regulate metabolism and intestinal function through prebiotic properties, and impact on gut microbiota.

\section{Acknowledgement}

The authors express their thanks to Organic Sp. z o. o. Polska for donating topinambur preparations.

\section{REFERENCES}

1. Yaeghoobi-Khanghahi F, Kazemi-Tabar SK, Gholipour A, Soorni J. GC-MS analysis of the methanolic extract of Jerusalem artichoke (Helianthus tuberosus) tubers. Int J Biosci. 2014; 5: 156-161.

2. De Santis D, Frangipane MT. Evaluation of chemical composition and sensory profile in Jerusalem artichoke (Helianthus tuberosus L) tubers: The effect of clones and cooking conditions. Int J Gastro Food Sci. 2018; 11: 25-30.

3. Yang L, He QS. Corscadden K, Udenigwe CC. The prospects of Jerusalem artichoke in functional food ingredients and bioenergy production. Biotechnol Rep. 2015; 5: 77-88.

4. Taha HS, El-Kawy AMA, Fathalla EK. A new approach for achievement of inulin accumulation in suspension cultures of Jerusalem artichoke (Helianthus tuberosus) using biotic elicitors. J Genet Eng Biotechnol. 2012; 10: 33-38.

5. Rosati A. Coltivate il topinambur, il sapore dei suoi tuberi ricorda quello del carciofo. Vita Camp. 2010; 10: 23-25.

6. Negro MJ, Ballesteros I, Manzanares P, Oliva JM, Sáez F, Ballesteros M. Inulin containing biomass for ethanol production: Carbohydrate extraction and ethanol fermentation. Appl Biochem Biotechnol. 2006; 132: 922-932.

7. Kaldy MS, Johnston A, Wilson DB. Nutritive value of Indian bread-root, squaw-root, and Jerusalem artichoke. Econ Bot. 1980; 34: 352-357.

8. De Leenheer L. Production and use of inulin: industrial reality with a promising future. In: Van Bekkum H, Roper H, Voragen F (eds) Carbohydrates as organic raw materials III. Wiley-VCH Verlag GmbH, Weinheim, 1996; 67-92.

9. Maicaurkaew S, Jogloy S, Hamaker BR, Ningsanond S. Fructan:fructan 1-fructosyltransferase and inulin hydrolase activities relating to inulin and soluble sugars in Jerusalem artichoke (Helianthus tuberosus Linn.) tubers during storage. J Food Sci Technol. 2017; 54(3): 698-706.

10. Saulnier DM, Ringel Y, Heyman MB, Foster JA, Bercik P, Shulman $\mathrm{RJ}$, et al. The intestinal microbiome, probiotics and prebiotics in neurogastroenterology. Gut Microbes. 2013; 4(1): 17-27.

11. Kareb O, Aïder M. Whey and Its Derivatives for Probiotics, Prebiotics, Synbiotics, and Functional Foods: a Critical Review. Probiotics and Antimicrobial Proteins. 2018; 1-22.

12. Alles MS, Hautvast JG, Nagengast FM, Hartemink R, Van Laere KM, Jansen JB. Fate of fructo-oligosaccharides in the human intestine. Brit J Nutr. 1996; 76: 211-221.

13. Rastall RA, Gibson GR, Gill HS, Guarner F, Klaenhammer TR, Pot B, Reid G, Rowland IR, Sanders ME. Modulation of the microbial ecology of the human colon by probiotics, prebiotics and synbiotics to enhance human health: an overview of enabling science and potential applications. FEMS Microbiol Ecol. 2005; 52: 145-152. 
14. Vogt L, Meyer D, Pullens G, Faas M, Smelt M, Venema K, et al. Immunological properties of inulin-type fructans Crit Rev Food Sci Nutr. 2015; 55: 414-436.

15. Shanzhao J, Ling L, Zhaopu L, Xiaohua L, Hongbo S, Jiayao C. Characterization of marine Pseudomonas pp. antagonist towards three tuber-rotting fungi from Jerusalem artichoke, a new industrial crop. Ind Crops Prod. 2013; 43: 556-561.

16. Panchev I, Delchev N, Kovacheva D, Slavov A. Physicochemical characteristics of inulins obtained from Jerusalem artichoke (Helianthus tuberosus L.). Eur Food Res Technol. 2011; 233: 889-896.

17. Praznik W, Cieslik E, Filipiak-Florkiewicz A. Soluble dietary fibers in Jerusalem artichoke powders: composition and application in bread. Nahrung. 2002; 46: 151-157.

18. Kim S, Kim CH. Evaluation of whole Jerusalem artichoke (Helianthus tuberosus L.) for consolidated bioprocessing ethanol production. Renew Energy 2014; 65: 83-91.

19. Pan L, Sinden MR, Kennedy AH, Chai H, Watson LE, Graham TL, Kinghorn AD. Bioactive constituents of Helianthus tuberosus (Jerusalem artichoke), Phytochem Lett. 2009; 2: 15-18.

20. Yuan XY, Gao MZ, Xiao HB, Tan CY, Du YG. Free radical scavenging activities and bioactive substances of Jerusalem artichoke (Helianthus tuberosus L.) leaves. J Food Chem. 2012; 133: 10-14.

21. Yuan XY, Cheng MC, Gao MZ, Zhuo RJ, Zhang LX, Xiao HB. Cytotoxic constituents from the leaves of Jerusalem artichoke (Helianthus tuberosus L.) and their structure-activity relationships. Phytochem Lett. 2013; 6: 21-25.

22. Liu HW, Liu ZP, Liu L, Zhao GM. Studies on the antifungal activities and chemical components of extracts from Helianthus tuberosus leaves. Nat Prod Res Dev. 2007; 19: 405-409.

23. Han R, Wang LH, Zhong QW, Sun K, Li Y. Study on antifungal activity of the extract from the leaves of Helianthus tuberosus. Mod Agric Sci Technol. 2010: 5.

24. Chen FJ, Long XH, Yu MN, Liu ZP, Liu L, Shao HB. Phenolics and antifungal activities analysis in industrial crop Jerusalem artichoke (Helianthus tuberosus L.) leaves. Indus Crops Prod. 2013; 47: 339-345.

25. Rubel IA, Pérez EE, Genovese DB, Manrique GD. In vitro prebiotic activity of inulin-rich carbohydrates extracted from Jerusalem artichoke (Helianthus tuberosus L.) tubers at different storage times by Lactobacillus paracasei. Food Res Int. 2014; 62: 59-65.

26. Hu JF, Qiu SY. Research process in ethanol production by the fermentation of Jerusalem artichoke. Liq.-Mark. Sci Technol. 2009; 182: 100-104.

27. Cheng Y, Zhou W, Gao CF, Lan K, Gao Y, Wu Q. Biodiesel production from Jerusalem artichoke (Helianthus Tuberosus L.) tuber by heterotrophic microalgae Chlorella protothecoides. J Chem Technol Biotechnol. 2009; 84:777-81.

28. Sung M, Seo YH, Han S, Han JI. Biodiesel production from yeast Cryptococcus sp. using Jerusalem artichoke. Bioresour Technol. 2014; 155: 77.

29. Sarchami T, Rehmann L. Optimizing enzymatic hydrolysis of inulin from Jerusalem artichoke tubers for fermentative butanol production, Biomass Bioenergy. 2014; 69: 175-182.

30. Chen L, Xin C, Deng P, Ren J, Liang H, Bai F. Butanol production from hydrolysate of Jerusalem artichoke juice by Clostridium acetobutylicum L7. Shengwu Gongcheng Xuebao/ Chin J Biotechnol. 2010; 26: 991e6.

31. Andersen AA, Greaves JE. d-lactic acid fermentation of Jerusalem artichokes. Ind Eng Chem. 1942; 24(12): 1522-6.
32. Ge X, Qian H, Zhang W. Improvement of l-lactic acid production from Jerusalem artichoke tubers by mixed culture of Aspergillus niger and Lactobacillus sp. Bioresour Technol. 2009; 100: 1872.

33. Wang L, Xue Z, Bo Z, Bo Y, Xu P, Ma Y. Jerusalem artichoke powder: a useful material in producing high-optical-purity l-lactate using an efficient sugar-utilizing thermophilic Bacillus coagulans strain. Bioresour Technol. 2013; 130: 174-80.

34. Huang J, Cai J, Wang J, Zhu X, Huang L, Yang ST, Xu Z. Efficient production of butyric acid from Jerusalem artichoke by immobilized Clostridium tyrobutyricum in a fibrous-bed bioreactor. Bioresour Technol. 2011; 102: 3923-6.

35. Liu XY, Chi Z, Liu GL, Wang F, Madzak C, Chi ZM. Inulin hydrolysis and citric acid production from inulin using the surface-engineered Yarrowia lipolytica displaying inulinase, Metab Eng. 2010; 12: 469-476.

36. Sridhar J, Eiteman MA. Influence of redox potential on product distribution in Clostridium thermosuccinogenes, Appl Biochem Biotechnol. 1999; 82: 91-101.

37. Wei W, Wu K, Qin Y, Xie Z, Zhu X. Intergeneric protoplast fusion between Kluyveromyces and Saccharomyces cerevisiae to produce sorbitol from Jerusalem artichokes, Biotechnol Lett. 2001; 23: 799-803.

38. Zaky EA. Physiological Response to Diets Fortified with Jerusalem Artichoke Tubers (Helianthus tuberosus L.) Powder by Diabetic Rats. Environ Sci. 2009; 5: 682-688.

39. Abdel-Hamid NM, Nazmy MH, Wahid A, Abdel-Moniem EM. Jerusalem artichoke attenuates experimental hepatic fibrosis via modulation of apoptotic signaling and fibrogenic activity. Biochemistry Biotechnol Res. 2015; 3: 43-50.

40. Wang PY, Kaneko T, Tsukada H, Nakano M, Nakajima T, Sato A. Time courses of hepatic injuries induced by chloroform and by carbon tetrachloride: comparison of biochemical and histopathological changes. Arch Toxicol. 1997; 71: 638-645.

41. Yang HJ, Kwon DY, Kim MJ, Kang S, Kim DS, Park S. Jerusalem artichoke and chungkookjang additively improve insulin secretion and sensitivity in diabetic rats. Nutr Metab (Lond) 2012; 9: 112.

42. Chang WC, Jia H, Aw W, Saito K, Hasegawa S, Kato H. Beneficial effects of soluble dietary Jerusalem artichoke (Helianthus tuberosus) in the prevention of the onset of type 2 diabetes and non-alcoholic fatty liver disease in high-fructose diet-fed rats. Br J Nutr. 2014; 112: 709-717.

43. Maicaurkaew S, Jogloy S, Hamaker BR, Ningsanond S. Fructan: fructan 1-fructosyltransferase and inulin hydrolase activities relating to inulin and soluble sugars in Jerusalem artichoke (Helianthus tuberosus Linn.) tubers during storage. J Food Sci Technol. 2017; 54(3): 698-706.

44. Samal L, Chaturvedi VB, Pattanaik AK. Effects of dietary supplementation with Jerusalem artichoke (Helianthus tuberosus L.) tubers on growth performance, nutrient digestibility, activity and composition of large intestinal microbiota in rats. J Anim Feed Sci. 2017; 26: 50-58.

45. Valdovska A, Jemeljanovs A, Pilmane M, Zitare I, Konosonoka IH, Lazdins M. Alternative for improving gut microbiota: use of Jerusalem artichoke and probiotics in diet of weaned piglets. Pol J Vet Sci. 2014; 17: 61-69.

46. Boonanuntanasarn S, Tiengtam N, Pitaksong T, Piromyou P, Teaumroong N. Effects of dietary inulin and Jerusalem artichoke (Helianthus tuberosus) on intestinal microbiota community and morphology of Nile tilapia (Oreochromis niloticus) fingerlings. Aquaculture Nut. 2018; 24: 712-722. 